\title{
EFFECT OF ADDING DIFFERENT LEVELS OF CITY WASTE COMPOST ON SOME PHYSICAL CHARACTERISTICS OF THE SOIL AND GROWTH OF Zea mays PLANTS .
}

Al-Magrebi, N. M.

Soil and Water Department, Faculty of Agriculture, Sana University, - Alyamen najeebalmagrebi@yahoo.com

تأثثير إضـافة مستويات مختلفة من سـماد ق قمامـة المدن على بعض خواص التربـة الفيزيائيسة ونمـو نبـات الذرة

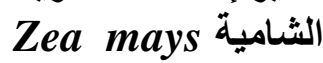

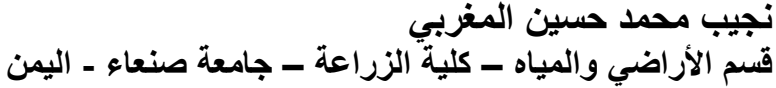

\section{الملخص}

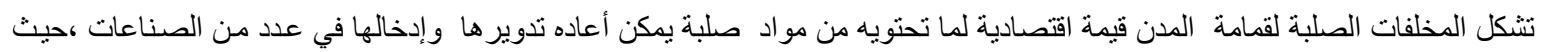

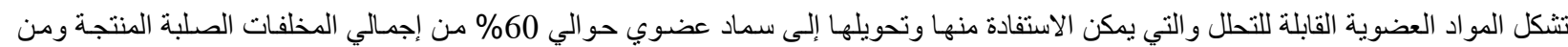

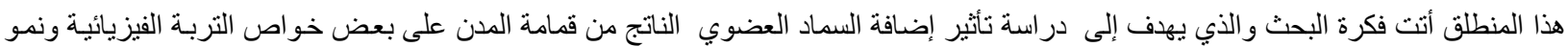

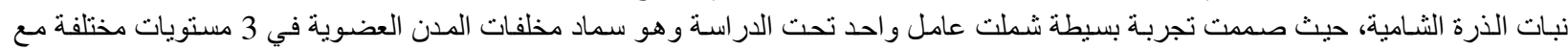
الكنترول هي 0، 10، 20، 40 طن/هكتار 0

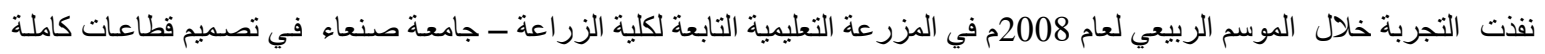

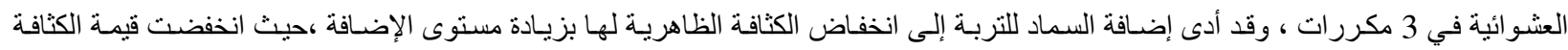

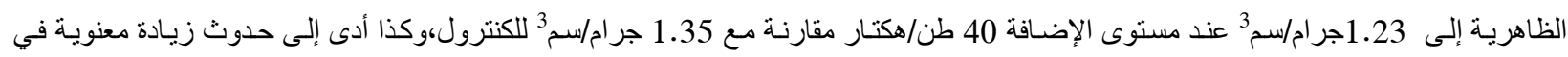

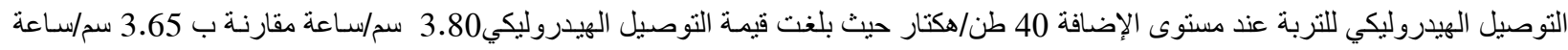

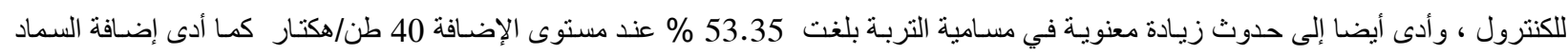
العضوي إلى حدوث زيادة معنوية في نسبة الماء الميسر للنبات حيث كانت نسبة الماء الميسر للنبات 8.73 8.

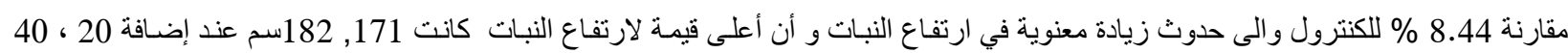

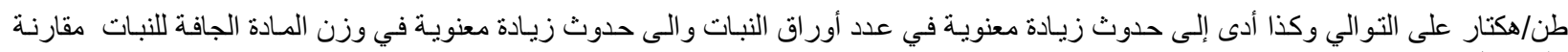
بالكنترول.

كلمات مفتاحيه:خواص التربة الفيزيائية ـ السماد العضوي ـ قمامة الددن - نبات الذرة 0

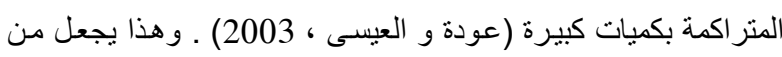

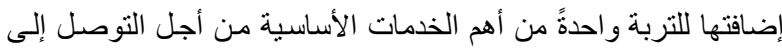

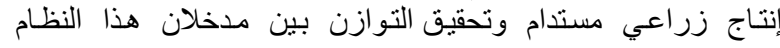

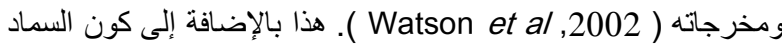

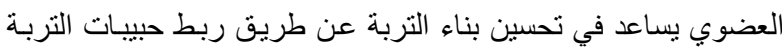

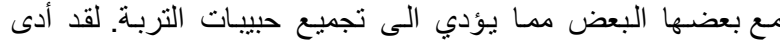

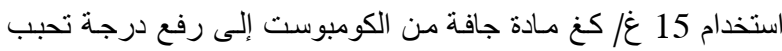

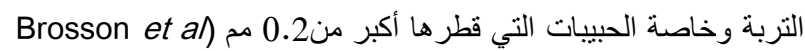

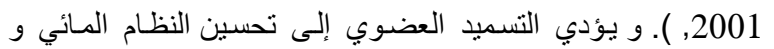

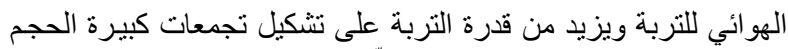

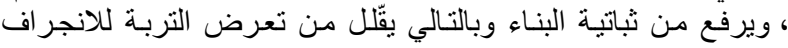

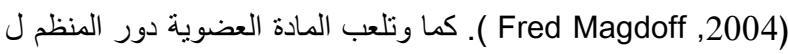

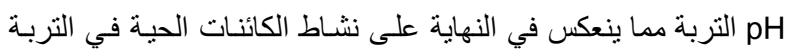

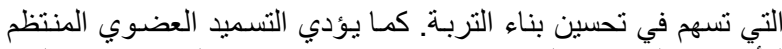

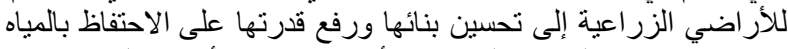

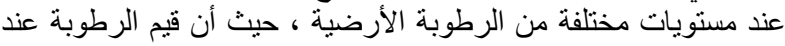

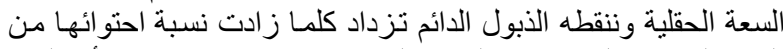

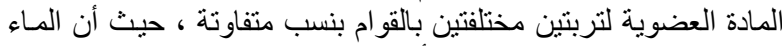

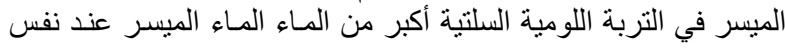

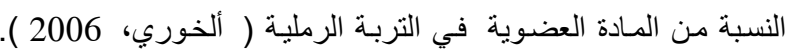

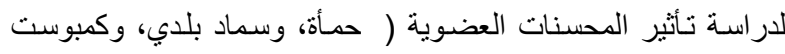

\section{المقدمة}

تعـاني معظم التـرب اليمنيـة مـن انخفـاض محتو اهـا مـن المـادة

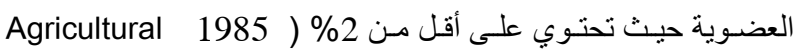
) و قد يعود ذلك إلى أن هذه المناطق تعتبر (Research Authority ,

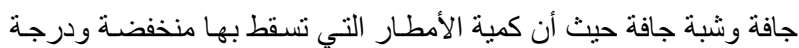

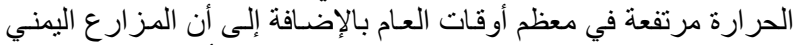

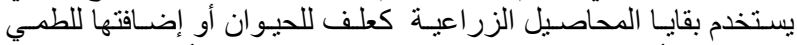

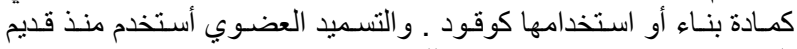

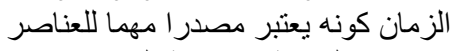

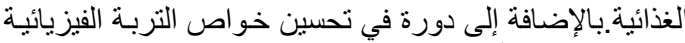

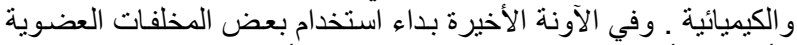

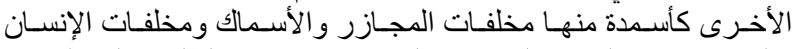

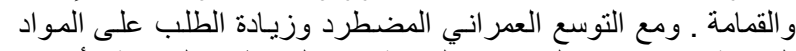

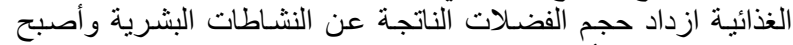

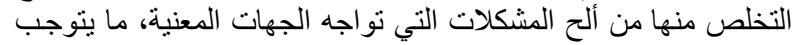

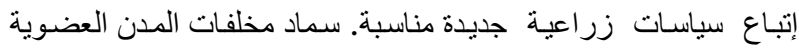

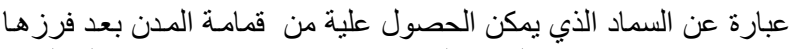

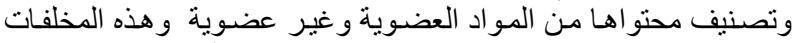

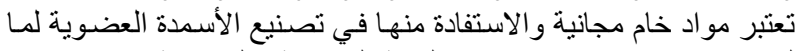
لها من فو ائد في تحسين خو اص التبن التربة الفيزيائية والكيميائية.

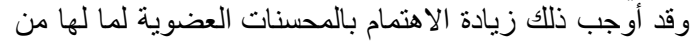

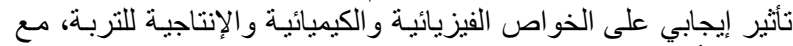

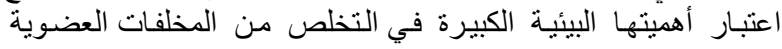


الصلبة للعاصمة صنعاء على بعض خو اص التربـة الفيزيائية ونمو نبات

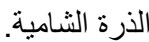
مواد وطر ائق العملة. - المل

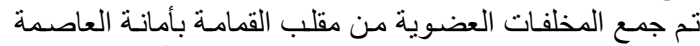

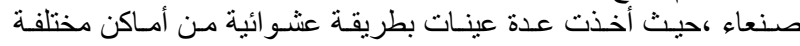

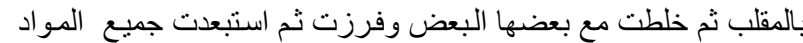

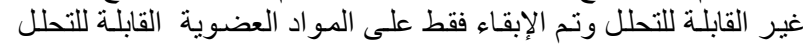

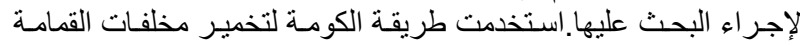

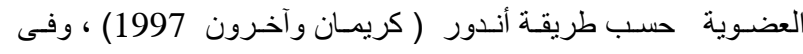

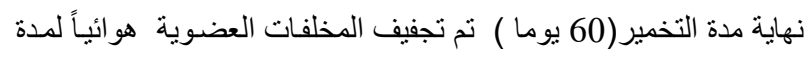

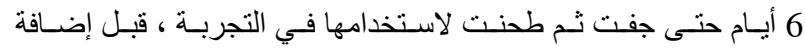

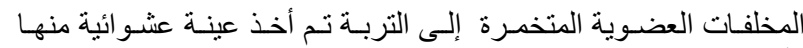
وأجريت عليها التقديرات المختلفة لمعرفة محتو اها من العناصر الغذائية

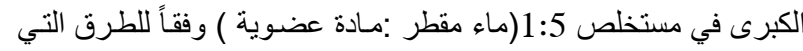

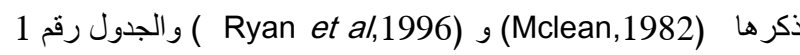
يبين الخواص الكيميائية للسماد العضوي المستخدم في التجربة.
القمامـة ) في بعض الخواص الفيزيائية للتربـة وفي إنتاجيـة محصول

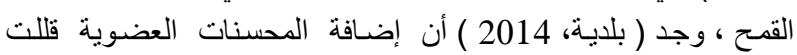

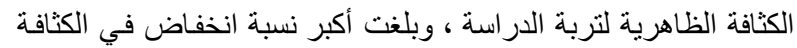

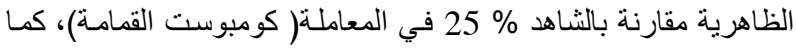

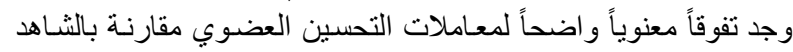

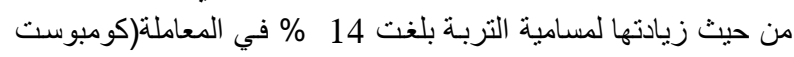

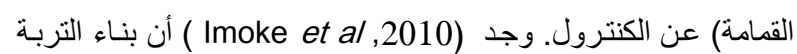
يعتبر من أهم العو امل المؤثرة في إنتاجيتها، ومن المكن أن أن بصبح

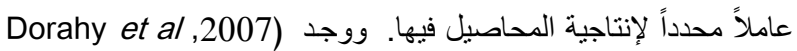

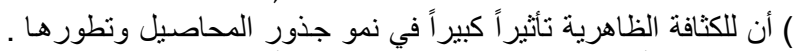

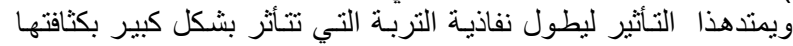

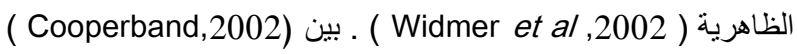
أن ارتفاع المسامية الكلية في الترب التي تلقت إضـافات من المـادة

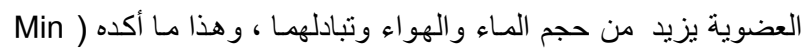

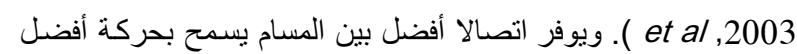

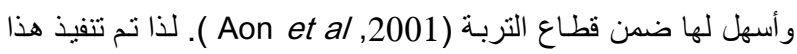

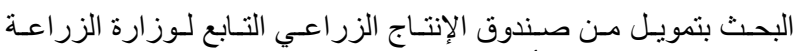

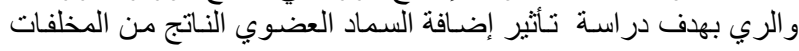

جدول 1 : بعض الخواص الكيميائية لسماد المخلفات العضوية (القمامة) المستخدم في التجربة.

\begin{tabular}{|c|c|c|c|c|}
\hline القيمة & الخاصية & القيمة & الوحدة & الخاصية \\
\hline 1.07 & النتروجين الكلي \% & 7.32 & & درجة التفاعل \\
\hline 20.74 & الكربون العضوي \% & 18.25 & مليمكافئ/لتر & الكالسيو م الذائب \\
\hline 35.92 & المادة العضوية \% & 12.60 & مليمكافئ/لتر & المغنيسيوم الذائب \\
\hline 19.38 & الكربون : النتروجين & 12.12 & مليمكافئ/لتر & الصوديوم الذائب \\
\hline 0.55 & الفسفور الكلي \% & 13.17 & مليمكافئ/لتر & البوتاسيوم الذائب \\
\hline 146.80 & الحديد ppm & 24.16 & مليمكافئ/لتر & الكلور ايد الذائب \\
\hline 84.20 & المنجنيز ppm & 0.06 & مليمكافئ/لتر & الكربونات الذائبه \\
\hline 52.11 & النحاس ppm & 7.40 & مليمكافئ/لتر & البيكربونات الذائبه \\
\hline \multirow[t]{2}{*}{96.41} & الزنك ppm & 22.14 & مليمكافئ/لتر & الكبريتات \\
\hline & & 6.61 & $\mathrm{dSm}^{-1}$ & 5: 1مستخلص \\
\hline
\end{tabular}

التعليميـة التابعة لكليـة الزر اعـة - جامعـة صنعاء في تصـميم قطاعـات

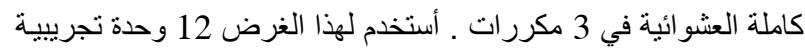

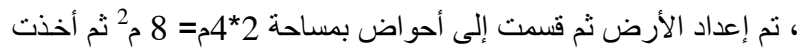
من التربة عينة عشو ائية تم تحليلها قبل الزراعة فيز لاعيائياً جدول 2.

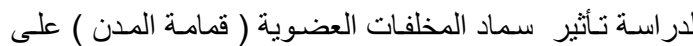

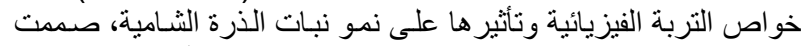

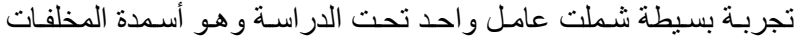

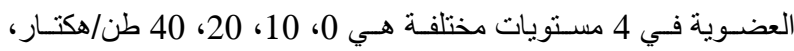

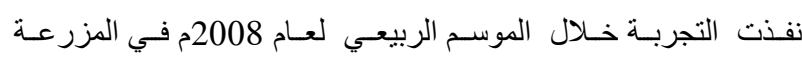

جدول 2 : الخواص الفيزيائية للتربة المستخدمة في التجربة

\begin{tabular}{|c|c|c|c|c|}
\hline القيمة & مفصولات التربة & القيمة & الوحدة & الخاصية \\
\hline 50 & الرمل \% & 1.36 & جر ام/سم3 & الكثافة الظاهرية \\
\hline 25 & السلت \% & 47.10 & $\%$ & المسامية \\
\hline 25 & الطين \% & 3.68 & سم/ساعة & التوصيل الهيدروليكي \\
\hline \multirow[t]{4}{*}{ Sandy Loam } & ق قوام التربة & 30.00 & $\%$ & النسبة المئوية للتشبع \\
\hline & & 16 & $\%$ & السعة الحقلية \\
\hline & & 8.20 & $\%$ & الماء الميسر للنبات \\
\hline & & 7.50 & $\%$ & نقطة الذبول \\
\hline
\end{tabular}




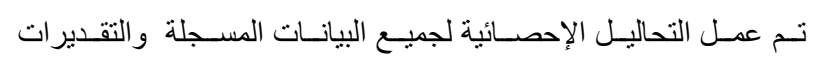
المدروسة تبعاً للطريقة الموصوفة من قبل(Steel and Torie,1960)

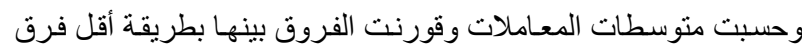

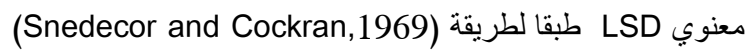

\section{النتائج والمناقشة الماتة}

تأثير إضافة سماد المخلفات العضوية(قمامة المدينة)على بعض خواص ولاصله التربة الفيزيائية إنيمان

تشير النتائج المتحصل عليها والموضحة في الثكل رقم 1 إلى

وجود فروق في الكثافة الظاهرية للتربة عند مستوى معنويـانية 0.05 نتيجة

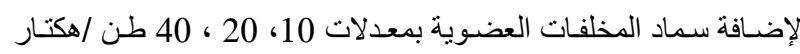

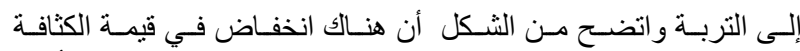

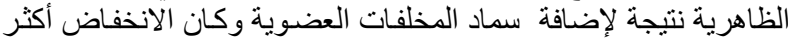

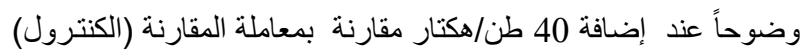

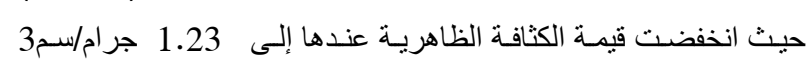
بالمقارنة ب 1.35 جر ام/سم3 للكنترول و على العكس من ذللك لم يكن

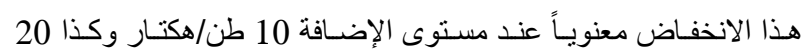
طن/هكتار حيث كانت الفروق بين كل منهم وبين الكنترول غير معنوية.

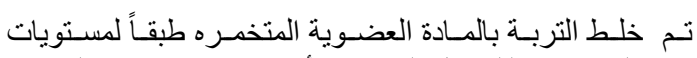

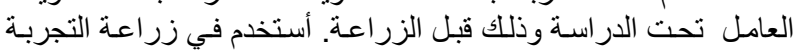

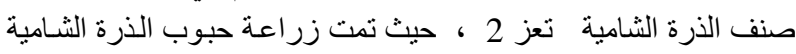

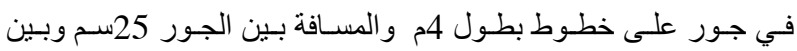
الخطوط 75سم وفقا لل (الساهوكي ،2000) .

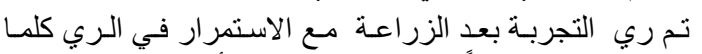

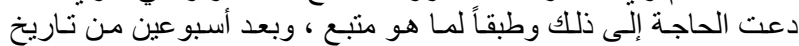

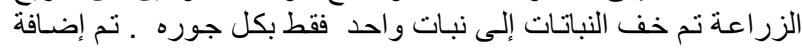

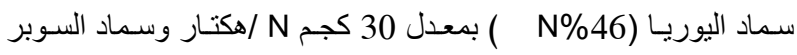

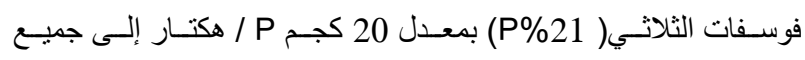

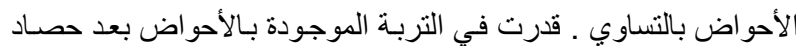

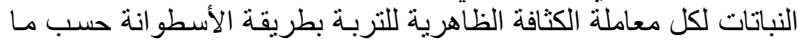

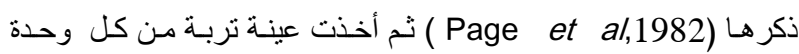

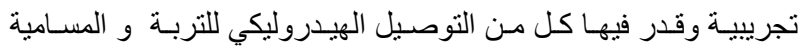

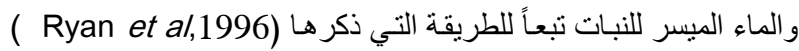

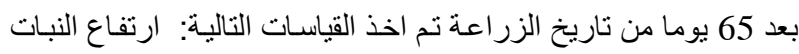

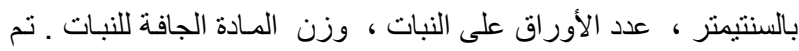

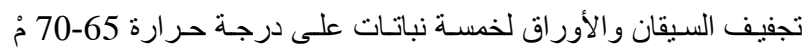

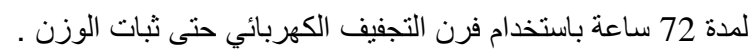

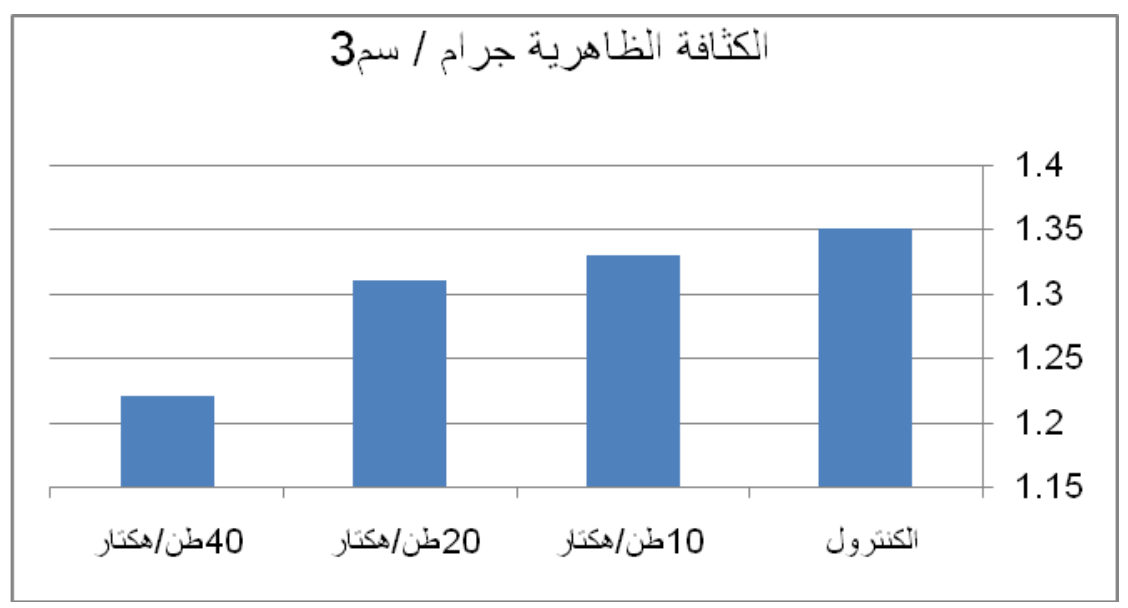

شكل1 : تأثير إضافة السماد العضوي على الكثافة الظاهرية للتربة

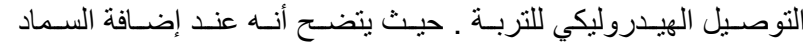
بالمستوى 40 طن/هكتار كانت الفروق بينها وبين الكنترول معنويـة ،

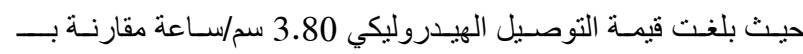
3.65 سم/ساعة للكنترول في حين لم تصل الزيـادة إلى درجـة المعنويـة

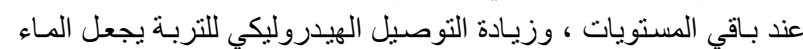

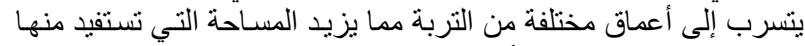

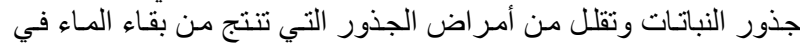

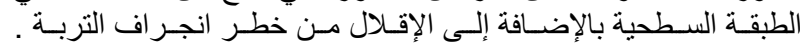

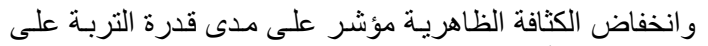

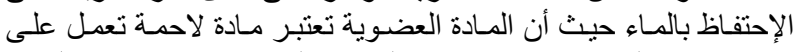

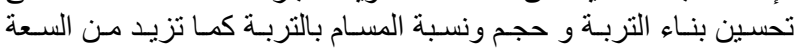

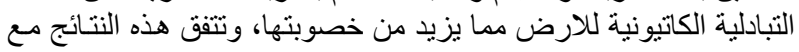

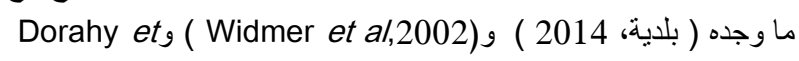

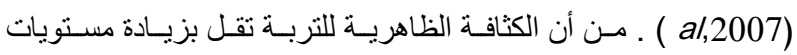
الإضافة للمخلفات العضوية.

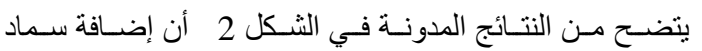

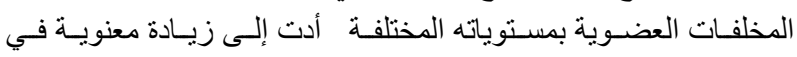


Al-Magrebi, N. M.

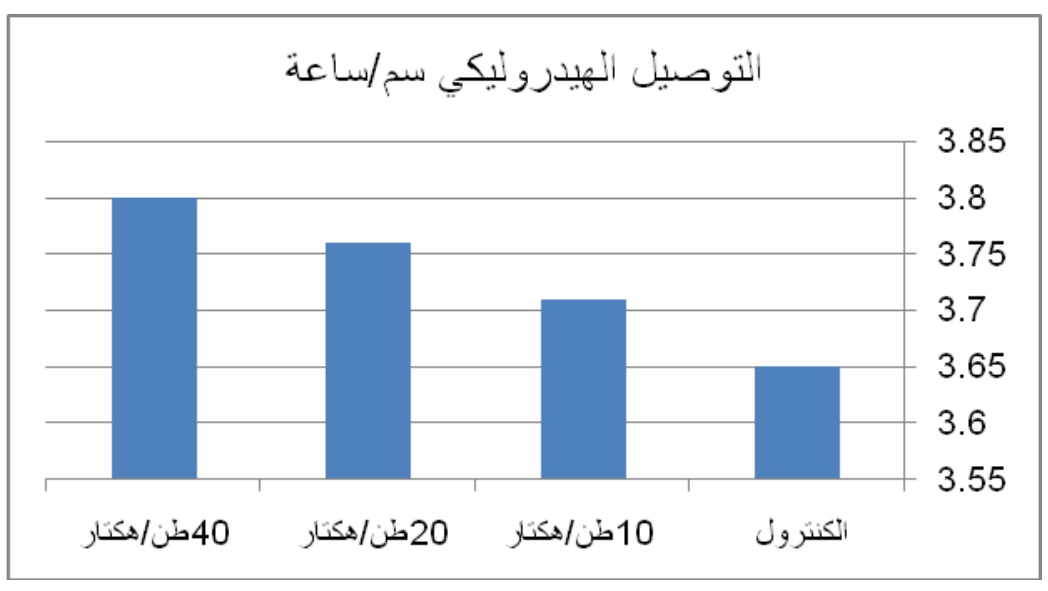

شكل 2: تأثير إضافة السماد العضوي على التوصيل الهياروليكي للتربة 
المعنويــة عنـــــــتوى الإضــافة المــخفض 10 طن/هكتــار مقارنــة

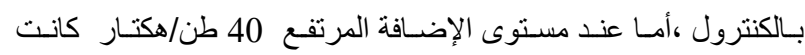

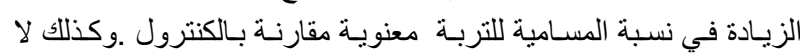

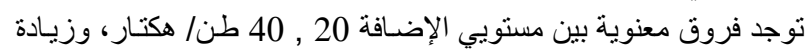

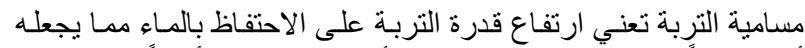

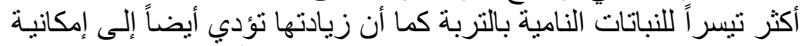

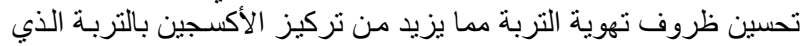

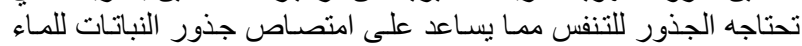
والعناصر الغذائية وينعكس ذللك على تحسين نمو وإنتاجية النبات .
و وتتفت هذه النتائج مع مـا وجده (Min ft al,2003 و Aon et

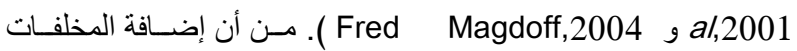

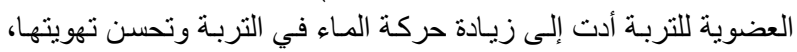

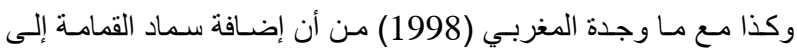

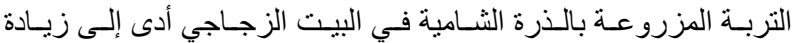

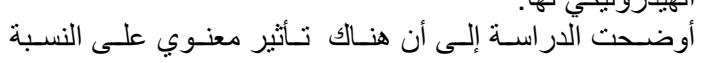
التوصيل الهيدروليكي لها.

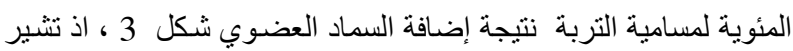

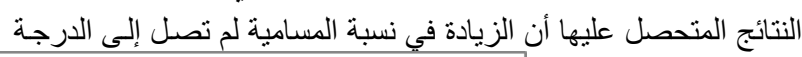

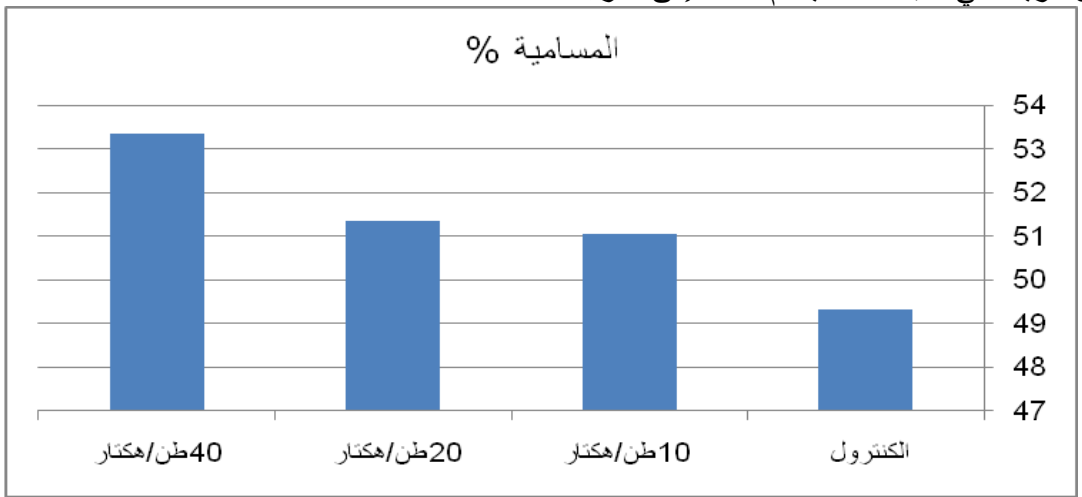

شكل3 : تأثير إضافة السماد العضوي على مسامية التربة

زادت نسبة المـاء الميسـر معنويـاً عند مستوى الإضـافة 40 طن/هكتـار

حيث كانت نسبة الماء الميسر للنبات 8.73 \% في حين كان الكنترول

، \%8.44

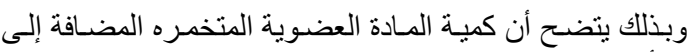

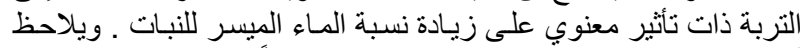

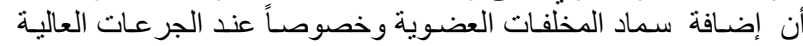

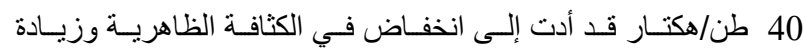

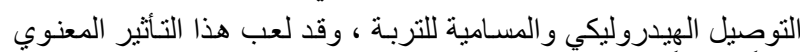

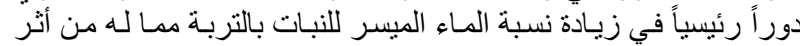

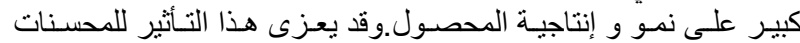

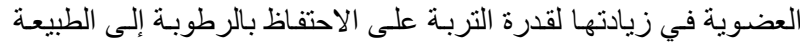

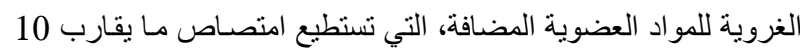

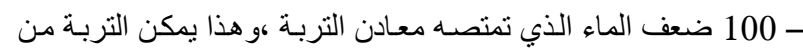

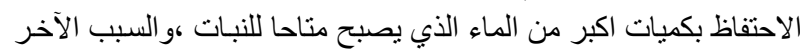

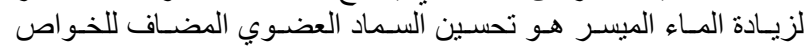

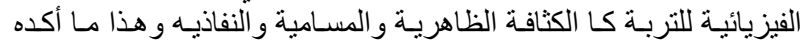

( Rizzi et al,2004)
و هذه النتيجة تتفق مع Wanas and Omran,2006 ) إذ إذ

تكون المسام بين جزيئات التربـة من النوع الصغير Micro pores أمـا

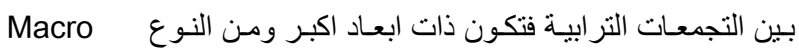

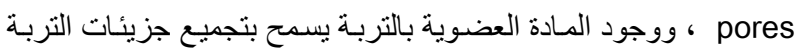

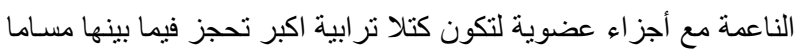

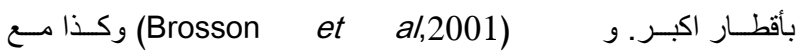

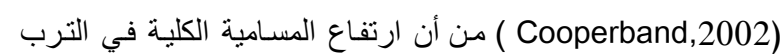

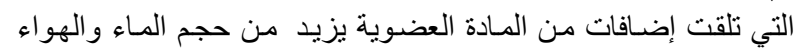

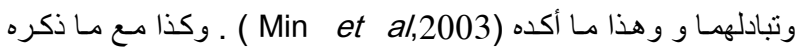
بلديـة( 2014 ) من وجود تفوقاً معنويـاً واضحاً لمعاملات التحسين

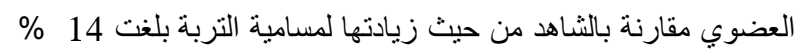
في المعاملة(كومبوست القمامة) عن الكنترول.

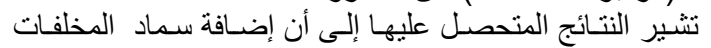

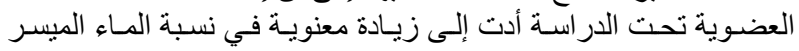
للنبات حيث تراوحت نسبة الماء الميسر في التربة من 8.46 و 8.73

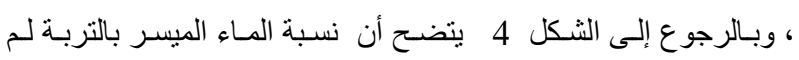

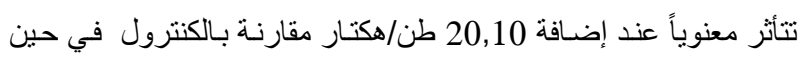


J.Soil Sci. and Agric. Eng., Mansoura Univ., Vol. 7 (2): 147 - 153, 2016

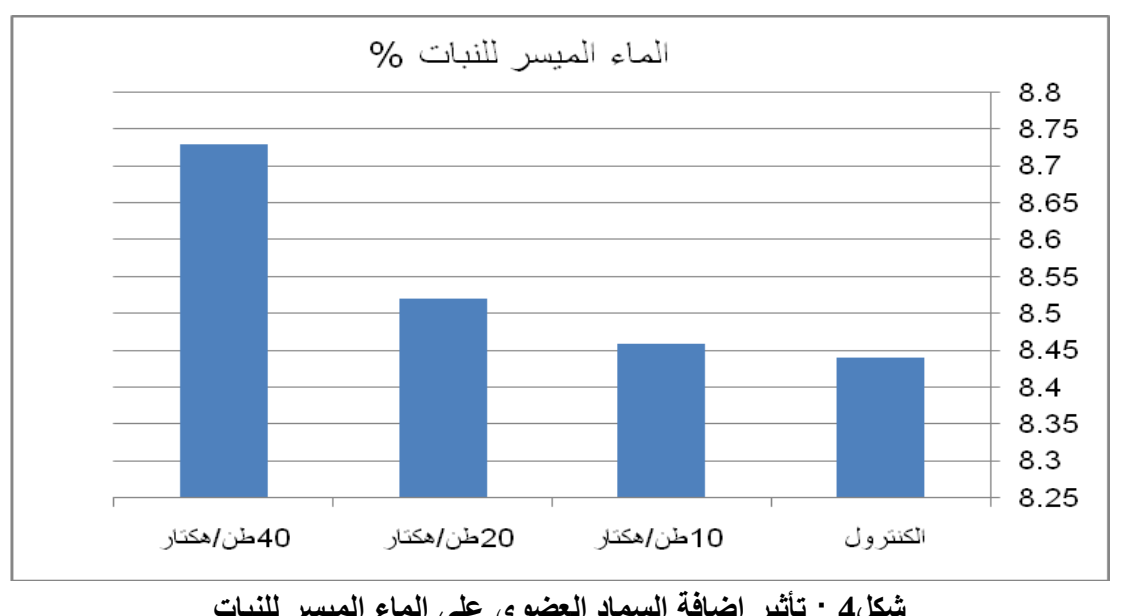

شكل4 : تأثير إضافة السماد العضوي على الماء الميسر للنبات 
و الكنترول من ناحية أخرى كانت معنويـة مـا عدا عند مستوى الإضـافة

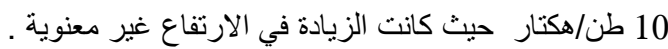

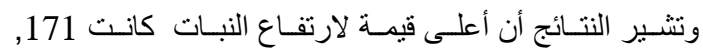
182سم عند إضافة 20 ، 40 طن/هكتار على التو الي ،وتتفق هذه النتائج

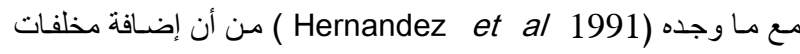
الأبقار إلى التربة أدت بصفة عامه إلى زيادة نمو نبات الذرة الثامية .
وتتفق هذه النتائج مع ما وجده ألخوري(2006) من أن التسميد

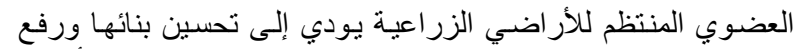
قدرتها على الاحتفاظ بالمياه عند مستويات مختلفة من الرية الرطوبة الرية الأرضية

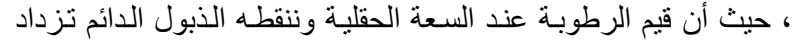

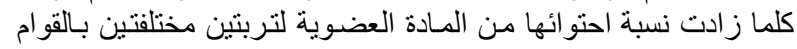

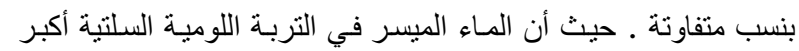

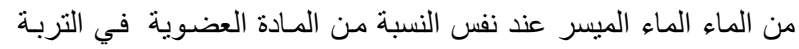

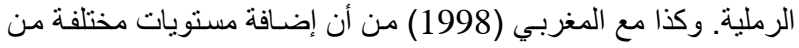

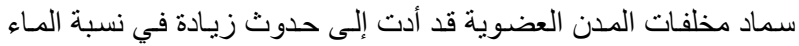

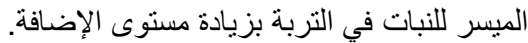

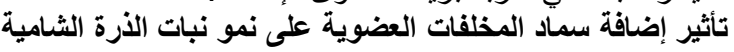

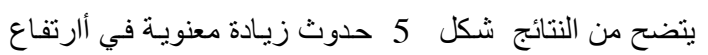

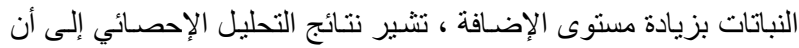

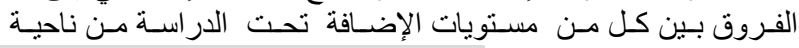

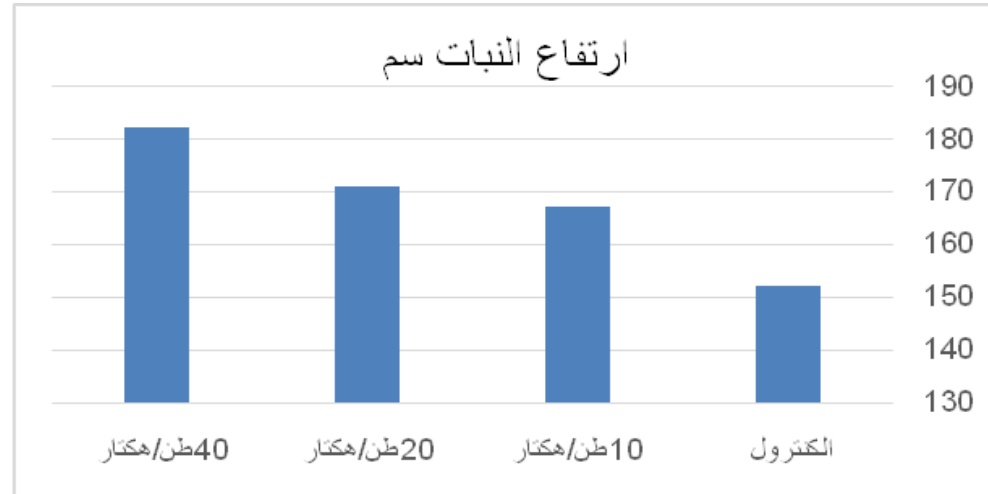

شكل 5: تأثير إضافة السماد العضوي على ارتفاع النبات سم
المـادة الجافـة عنــد إضـافة 20، 40 طـن / هكتـار كانــ 19.3 \% و 30 على الترتيب وذلك مقارنة بالكنترول ـوتنعد الخواص الفيزيائية

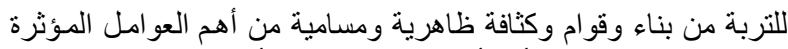

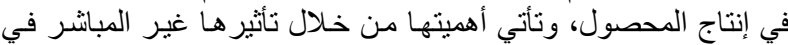

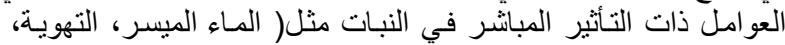

الحرارة.....إلخ ) (Letely et al,1985 ).

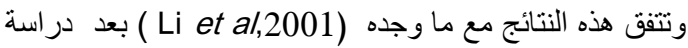

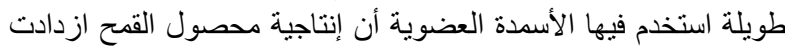

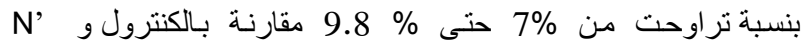

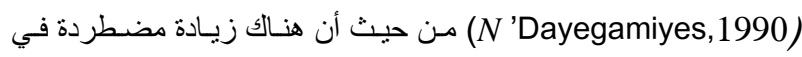

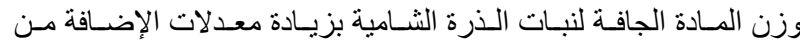
المخلفات العضوية بصفة عامة. لمان.
توضتح النتائج في الثكل 6 أن هنـاك فروق معنويـة في عدد

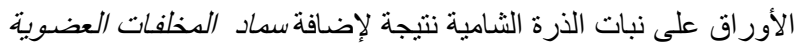

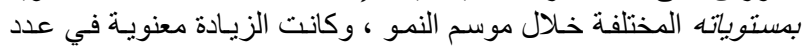

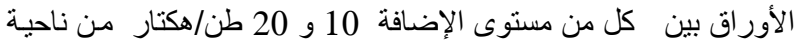

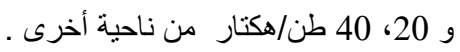

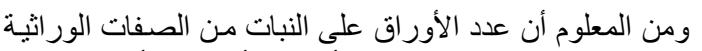

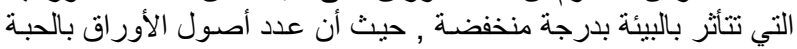

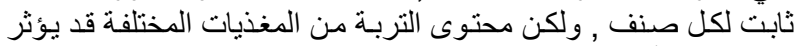

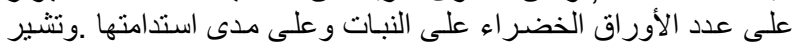
النتائج أن أقصى زيـادة في عدد الأوراق على النى النبات كانت بإضـافة 40

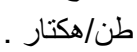

بالرجوع إلى الثكل 7 يلاحظورجود فروق عاليـة المعنويـة

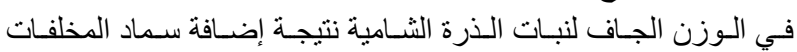

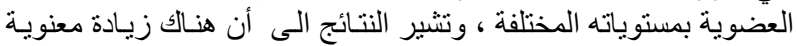

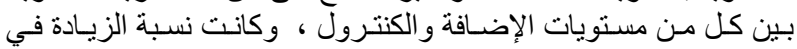




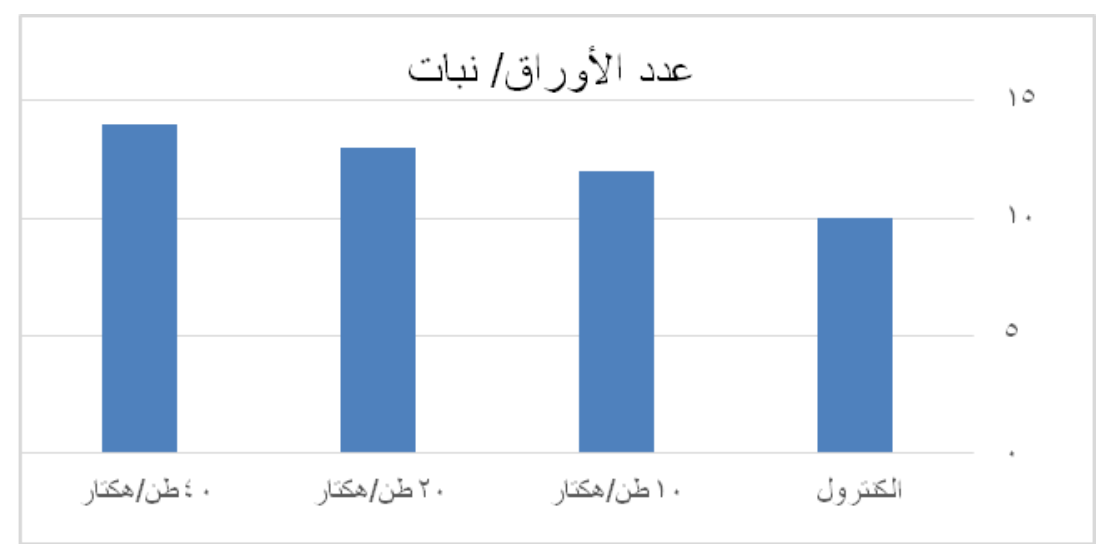

شكل 6: تأثير إضافة السماد العضوي على عدد الأوراق

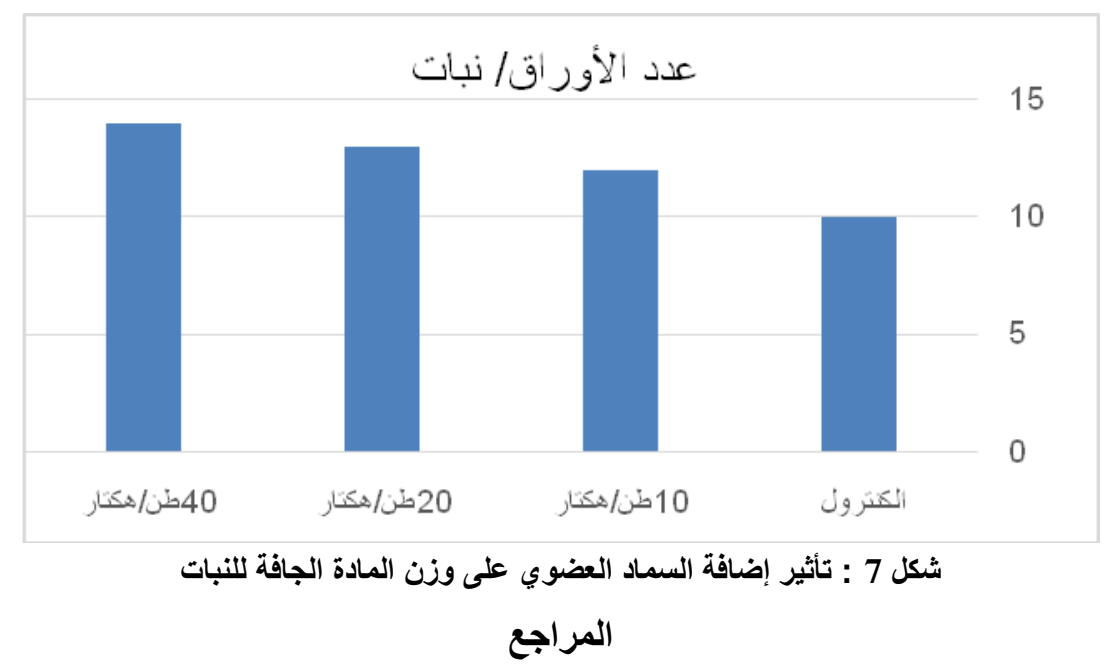

المغربي، نجيب محمد حسين. 1998. تأثير إضـافة المخلفات العضوية

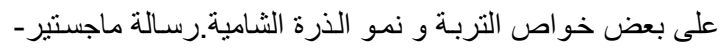

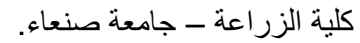

عودة ، محمود و العيسى عبد الله .2003 ـ تأثير استخدام أنواع مختلفة

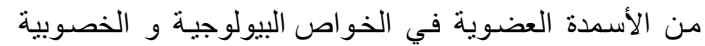

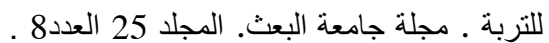

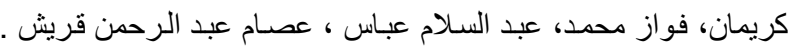

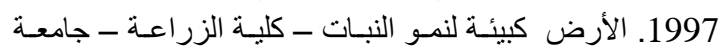

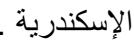

Agricultural Research Authority, (1985). Annual Report, 1984. Ministry of Agricultural and Fishers. Republic of Yemen, Taiz.

Aon, M. A.; D. E. Sarena. J. L; Burgos and S. Cortassa( 2001). Microbiological.Chemical and physical properties of soils subjected to conventional or no-till management: an assessment of their quality status, Soil Till.Res., 60:173-186.

Brosson , L; M, Koch, Y, Le Bissonnais, E Barriuso, and V,Lecomte( 2001). Soil surface structure stabilization by municiple waste compost application. Soil Sci. Soc. Am. J. no.65, p.18041811

Cooperband, L(2002). Building soil organic matter with organic amendments, Center of Integrated Agricultural Systems, 6-12.
بلدية ، رياض.2014.تحسين الخواص الفيزيائية للتربة باستخدام بعض

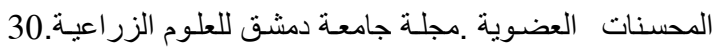

.39-27:(4)

الخوري ، عصـام. 2006 ـ أثر إضـافة معدلات مختلفة من الأسمدة

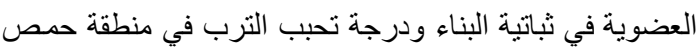
زيدل مجلة جامعة البعث - 28 (5 (5):143-154.

الساهوكي ، مدحت مجيد .2000. ارشادات في زر اعة الذرة الصفر اء مركز اباء للأبحاث الزر اعية - بغداد - العراق.

Dorahy, C. G., A. D. Pirle. P. Pengelly. L. M. Muirhead and K. Y. Chan.(2007).Guidelines for using compost in land rehabilitation and catchmentmanagement, Final report prepared for the Department of EnvironmentConservation (NSW).

Fred Magdoff, Ray, R. Weil (2004). Soil Organic Matter in Sustainable Agrigulture, CRC, Press, Boca Raton, New York, Washington D.C.

Hernandez, T.; Moreno, J.I. and Costa, F.(1991) .Influence of sewage sludge application on crop yields and heavy metal availability .Soil Science and Plant Nutrition 37 (2): 201-210

Imoke, E. D., U. J. Ibu. O. C. Omonya. O. J. Nwabueze. and G. N. Njar. (2010).Effects of land degradation on soil productivity in calabar south local 
government area, Nigeria, European. J. Soil .Sci. 18: $166-170$.

Letely, J.(1985). Relationship between soil physical properties and crop production, Adv. Soil. Sci., 1: 277-294.

Li, Z., J. A. Ryan. J. L. Chen and S. R. ALAbed.(2001). Adsorption of cadmium on bio solids amendments soils . J. Envir. Qual., 30: $903-$ 911.

Mclean, E.o (1982). Soil $\mathrm{pH}$ and lime requirement. P. 199-224, In: A. L. page (ed) Methods of Soil Analysis,m part2: Chemical and Microbiological Properties. Am. Soe. Agron, Madison, Wisc. U. S. A.

Min, D. H., K. R. Islam. L. R. Vough. and R. R. Weil. (2003). Dairy manure effects on soil quality properties and carbon sequestration in alfalfa orchard grass systems, Commun Soil Sci. Plant Anal., 34: 781- 799 .

N'Dayegamiye, A(1990). Effects of long term application of solid cattle manure on silage maize production and soil chemical properties .Canadian Journal of Plant Science. 70(3):767-775.

Page.,A.L.R.H.Miller,and D.R.Keency(1982).Methodds of Soil Analysis. part2 .Chemical and Microbiological Properties. Second Edition. Agronomy No.9.Amer.Soc.U.S.A.
Rizzi, L., G. Petruzzelli. and G. Vigna (2004). Soil physical changes and plant availability of $\mathrm{Zn}$ and $\mathrm{Pb}$ in a treat ability test of phyto -Stabilization chemosphere, 57: 1039 - 1046.

Ryan , J., S. Garabet, K.Harmsen , and A.Rashio(1996). A Soil and Plant Analysis Manual Adapted for the West Asia and North Africa . Region .ICARDA , Allippo, Syria , 140pp

Snedecor, G.M. and Cochran, W.C. (1969) Statistical Methods. 6th Edition, Iowa University Press. Ames, Iowa

Steel, R.G.D. and Torrie,J.H.(1960). Principles and Procedures of Statistics Mc Graw . Hill Book company Inc. Newyork.

Wanas, Sh. and W. Omran(2006). Advantages of applying various compost types to different layers of sandy soil: 1- Hydro - physical properties. J.App. Sci . Rec., 2(12): 1298- 1303.

Watson, C. A., H. Bengtsson. A. K. Loes. A. Myrbeck. E. Alomon. J. Schroder and E. A. Tockolale(2002). A review of farm scale nutrient budgets for organic farms as $\mathrm{s}$ tool for management of soil fertility. Soil Use and Management, 18: 264 - 273.

Widmer, T. L., N. A. Mitkowski and G. S. Abawi(2002). Soil organic matter and Management of plant - parasitic nematodes. J. Nematology, 4:289-295

\title{
EFFECT OF ADDING DIFFERENT LEVELS OF CITY WASTE COMPOST ON SOME PHYSICAL CHARACTERISTICS OF THE SOIL AND GROWTH OF ZEA MAYS PLANTS .
}

Al-Magrebi, N. M.

Soil and Water Department, Faculty of Agriculture, Sana University, - Alyamen najeebalmagrebi@yahoo.com

\begin{abstract}
The solid wastes of city represent an economic value for its free raw materials included which can be recycled and accessed into a number of industries in which organic materials could be composted and transferred to organic fertilizer represent $60 \%$ from the total solid remnants. So that the idea of the current study is aiming to study the impact of adding an organic fertilizer extracted from city garbage on some characteristics of the physical soil . A simple experiment has been designed including a lonely factor under studying which is the city waste compost fertilizer of the organic remnants at four different levels with control : 0,10,20,40 tons /hectares . The experiment was carried out during the spring season of 2008 at the Educational Farm , Faculty of Agriculture, Sana'a University . A complete randomized block design was used in three replicates. The addition of organic fertilizer led to reducing the bulk density of soil and increasing the addition rate. The Bulk Density value reduced to $1.23 \mathrm{~g} / \mathrm{cm}^{3}$ at added 40 tons /ha compared with $1.35 \mathrm{~g} / \mathrm{cm}^{3}$ for control, and has also led to a significant increase in the Hydraulic Conductivity of the soil at the level of added $40 \mathrm{t} / \mathrm{ha}$. as the value of hydraulic conductivity of $3.80 \mathrm{~cm} /$ hour , compared with $3.65 \mathrm{~cm} /$ hour to control . and has also led to a significant increase in soil porosity was $53.35 \%$ at added $40 \mathrm{t} / \mathrm{ha}$. also resulted a significant increase in the proportion of available water, where the percentage of available water was $8.73 \%$ at the level of 40 tons / ha compared to $8.44 \%$ for the control. and caused. A significant increase in plant height was observed , where the values of plant height were $171 \& 182 \mathrm{~cm}$ when add $20 \& 40 \mathrm{t} / \mathrm{ha}$, respectively, and also significant increases in both the number of leaves per plant dry matter weight per plant compared to the control .
\end{abstract}

Keywords : Physical characteristics of soil - organic fertilizer - city wastes compost - maize plants . 